\title{
Um método para estimativa de metas DEA
}

\author{
Ernée Kozyreff Filho \\ Engenheiro de Produção - Pilkington \\ E-mail:ernee@bol.com.br \\ Armando Zeferino Milioni \\ Professor Adjunto do Instituto Tecnológico de Aeronáutica \\ E-mail:milioni@ita.br
}

\section{Resumo}

A avaliação da produtividade de unidades comerciais pertencentes a um mesmo grupo, como as agências de um banco ou as unidades de uma rede de franquia, feita através de Análise de Envoltória de Dados (DEA, da sigla em inglês Data Envelopment Analysis], fornece valores de eficiência de cada uma destas unidades. Esta eficiência, no entanto, é relativa, pois seu valor depende de como as outras unidades se comportam. Como a análise é feita pósprodução, fica impossível saber quanto determinada unidade deveria ter produzido para ser classificada como eficiente. Este trabalho sugere um método de se estimar metas de produção para que cada unidade possa ser considerada eficiente independentemente do resto do grupo, a partir da idealização de uma fronteira de eficiência.

Palavras-chave

Pesquisa operacional, avaliação, DEA, eficiência, metas.

\section{A method for estimating goals in DEA}

\begin{abstract}
The productivity evaluation of commercial units belonging to a single group, such as the branches of a bank or the units of a franchising network, done through Data Envelopment Analysis (DEA), yields efficiency values for each of the units, called DMU's (Decision Making Units). However, this efficiency is relative, for its value depends on how the other DMU's behaved. Since the analysis is done after production, it is not possible to know how much one unit should have produced to be classified as efficient. This work suggests a method for estimating goals of production for each DMU to be considered efficient independently from the rest of the group, from the idealization of an efficiency frontier.
\end{abstract}

Key words

Operations research, evaluation, DEA, efficiency, goals. 


\section{INTRODUĈ̣̃O}

Uma das preocupações de corporações que possuem diversas unidades com diferentes características é saber como avaliar a eficiência destas unidades, visto que há muitos fatores que influenciam a produtividade de cada uma delas. Consideremos, por exemplo, uma rede de supermercados que atua em diversas cidades e cujas lojas possuem características semelhantes. É razoável supor que elas terão diferentes receitas, dado que atuam em mercados distintos. População, nível de renda, perfil do consumidor, localização, dentre outros, são fatores que afetam as vendas de cada loja.

Nesta última década, em especial, observamos a expansão das grandes redes comerciais que invadiram cidades, instalando nelas suas filiais. Os shopping centers possuem cada vez mais lojas e lanchonetes franqueadas. Academias e até lavanderias já têm marca e a tendência é que o número de firmas individuais diminua. Portanto, vemos que a questão da avaliação dessas unidades comerciais (doravante DMU's, sigla em inglês para Decision Making Units) é atual e de grande relevância.

Em 1978, Charnes, Cooper e Rhodes propuseram uma metodologia que visava fazer uma avaliação justa do ponto de vista matemático. Esta metodologia, chamada Análise de Envoltória de Dados, ou simplesmente DEA (da sigla em inglês, Data Envelopment Analysis), calcula, através de um problema de programação linear, a eficiência relativa de cada DMU. O modelo desenvolvido no artigo referenciado ganhou fama e ficou conhecido pelas iniciais dos autores, i.e., modelo CCR. Mais tarde, em 1984, Banker, Charnes e Cooper propuseram um modelo alternativo que ficou conhecido como modelo BCC. Os modelos CCR supõem retornos constantes de escala, enquanto nos modelos BCC os retornos de escala são variáveis. Os fundamentos de DEA podem ser encontrados nos livros de Charnes, Cooper, Lewin e Seiford (1994) e de Cooper, Seiford e Tone (2000).
O método é aplicável quando são fornecidos numericamente todos os Insumos e Produtos de cada DMU. Assim, é preciso, em primeiro lugar, decidir quais são esses Insumos e Produtos, o que pode não ser tão simples. Outra etapa necessária e nem sempre simples é conseguir os dados desejados. Inúmeros fatores podem dificultar sua obtenção, desde a não permissão para acesso até a pura falta dos mesmos.

\section{A metodologia DEA calcula a eficiência relativa de cada unidade de um grupo de unidades de produção}

Vencidos estes obstáculos, o método DEA calcula eficiências relativas para todas as DMU's através da resolução de um problema de otimização [SILVA, 1999].

Notemos, entretanto, que as DMU's não dispunham da informação sobre quanto deveriam produzir para que fossem consideradas eficientes. Deveriam, é claro, ter feito o melhor possível com seus recursos. Porém, é fácil aceitar que uma meta numérica seria de grande valia na concepção de melhores diretrizes aos seus esforços.

Seja, por exemplo, o caso de um banco que projeta para o ano seguinte um crescimento de $5 \%$ em uma determinada carteira de investimentos. Suponhamos que isto seja possível somente com a conquista de 10.000 novos clientes. Desta maneira, é preciso saber quantos clientes cada agência deve conquistar. Dizer simplesmente para que cada uma consiga o máximo possível não é tão estimulante nem objetivo como ter um número individual a alcançar.

É nesse contexto que situamos o presente trabalho. Outros autores têm pesquisado problemas semelhantes e uma boa referência recente nesse sentido é a de Lins et al., (2003). Contudo, não temos conhecimento da existência de outro trabalho cuja abordagem esteja baseada no estabelecimento de uma hipótese a respeito do lugar geométrico dos pontos que define a fronteira de eficiência, que

Tabela 1: Dados das lojas.

\begin{tabular}{|l|c|c|c|r|r|r|r|}
\hline \multicolumn{1}{|c|}{ LOJA } & A & B & C & D & E & F & G \\
\hline Funcionários & 2 & 3 & 4 & 2 & 6 & 5 \\
\hline Aluguel & 4 & 2 & 2 & 3 & 1 & 1 & 2 \\
\hline Vendas & 10 & 18 & 16 & 13 & 12 & 15 & 28 \\
\hline Vendas / Funcionário & 5,0 & 6,0 & 4,0 & 6,5 & 2,0 & 3,0 & 4,0 \\
\hline Vendas / Aluguel & 2,5 & 9,0 & 8,0 & 4,3 & 12,0 & 15,0 & 14,0 \\
\hline
\end{tabular}


é o que fazemos no presente trabalho numa formulação do tipo CCR com um único Produto.

\section{FUNDAMENTOS DE ANÁLISE DE ENVOLTÓRIA DE DADOS}

Seja um grupo hipotético de 7 lojas cujos Insumos sejam o número de funcionários e o valor do aluguel do prédio. Como Produtos, serão consideradas as vendas de cada unidade. A Tabela 1 mostra os valores de Insumos e Produtos para cada uma das lojas.

Uma maneira de visualizar melhor estes dados é colocando-os em um gráfico, que é o que fazemos na Figura 1. As coordenadas horizontais referem-se a Vendas/Funcionário, enquanto as verticais são relativas a Vendas/ Aluguel.

As lojas mais eficientes são aquelas que melhor transformam Insumos em Produtos e, portanto, possuem as maiores relações Produtos/Insumos. No entanto, há lojas que, apesar de não haverem obtido os valores máximos numa das produtividades, possuem valores relativamente altos nos dois quesitos.

A questão pode ser vista da seguinte maneira: suponhamos que fosse possível criar lojas a partir de uma combinação linear das lojas $D$ e $F$, que são as que obtive- ram os maiores índices de produtividade nos dois quesitos. Na Figura 1, essas lojas virtuais ficariam sobre o segmento de reta que liga os pontos $D$ e $F$, linha que definiria a fronteira de eficiência desse grupo de lojas. Como as lojas $B$ e $G$ estão a nordeste (mais à direita e acima) desta reta, elas definem uma nova fronteira de eficiência.

Estas 4 lojas $(B, D, G$ e $F)$ possuem, portanto, eficiência relativa $100 \%$. Isto não significa, porém, que não possam ser melhoradas [ALVES, 2001]. Apenas nos dizem que, para estes dados, são as que melhor transformam Insumos em Produtos, razão pela qual essa fronteira de eficiência também é conhecida como a fronteira do conjunto de possibilidades de produção [CHARNES et al., 1994].

Uma loja que deseje entrar para o grupo eficiente deve diminuir Insumos e/ou aumentar Produtos, o que na literatura corresponde a modelar o problema com orientação para os Insumos ou para os Produtos (em inglês, Input Oriented ou Output Oriented Models, respectivamente) [CHARNES et al., 1994].

Na Figura 1, se a loja diminuir o número de funcionários, estará caminhando horizontalmente para a direita, pois somente o valor da razão Vendas/Funcionário será afetado. Se apenas aumentar as vendas, o caminho per-

Figura 1: Comparação entre lojas - Dois Insumos e um Produto.

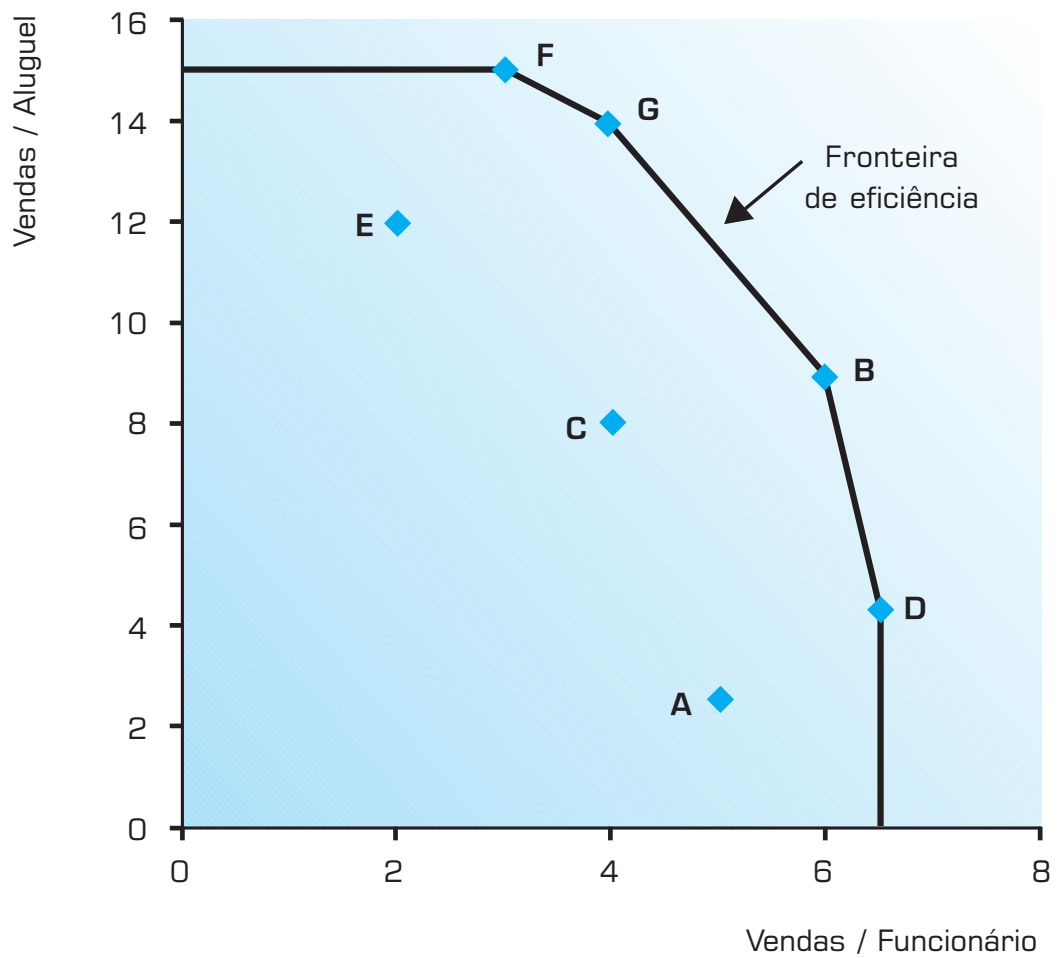


corrido pelo ponto será uma continuação da reta que o liga à origem, já que ambas as razões, Vendas/Funcionário e Vendas/Aluguel, serão afetadas na mesma proporção. Neste caso particular, o valor do aluguel pode não ser alterável (se estiver em um contrato, por exemplo). Caso essa alteração fosse factível, o ponto caminharia verticalmente. São comuns os casos em que nem todos os Insumos e Produtos podem ser variados [AVELLAR et al., 2002].

Para calcular a eficiência relativa das lojas ineficientes suporemos que os Insumos são fixos. Se somente os Produtos são variáveis, a única maneira de uma loja migrar para a fronteira de eficiência é vendendo mais.

Se essa migração se der sobre a reta que liga o ponto referente à loja à origem, a eficiência, então, pode ser medida pelo quociente entre a distância inicial desse ponto à origem e a distância da origem até o ponto que a loja almeja alcançar, que fica na fronteira de eficiência.

Com o auxílio da Figura 2 ilustramos o cálculo para a loja $C$ cuja eficiência será:

$$
\frac{O C}{O C},=78 \%
$$

Esse conceito de eficiência é também conhecido como eficiência técnica e indica que a loja $C$ está vendendo
$78 \%$ do que deveria estar vendendo para ser considerada eficiente dentro deste grupo de lojas. Ela está sendo comparada, na verdade, a uma loja virtual gerada por uma combinação linear entre $B$ e $G$. Podemos dizer que estas são as lojas eficientes do grupo que possuem um mix de Insumos parecido com o de $C$. São os seus benchmarks, ou o seu conjunto de referência.

Há outros conceitos de eficiência, como os conceitos de eficiência produtiva, ou de escala [CHARNES et al., 1994] cuja discussão foge ao escopo deste trabalho.

A Tabela 2 apresenta as eficiências das lojas do exemplo.

\section{METAS DEA}

Estabelecer a meta de um Produto para uma DMU significa saber qual é a porção de uma meta global para o conjunto de todas as DMU's que deve ser atribuída a esta DMU em particular. Esta meta, se atingida, deve conferir a ela o status de eficiente.

Neste trabalho propomos um método de distribuição de uma meta global para uma rede de DMU's de modo que, se todas elas atingirem suas metas, então todas elas formarão uma fronteira de eficiência convexa previamente idealizada por uma função no $\mathfrak{R}^{n}$. Esta função

Figura 2: Eficiência da loja C.

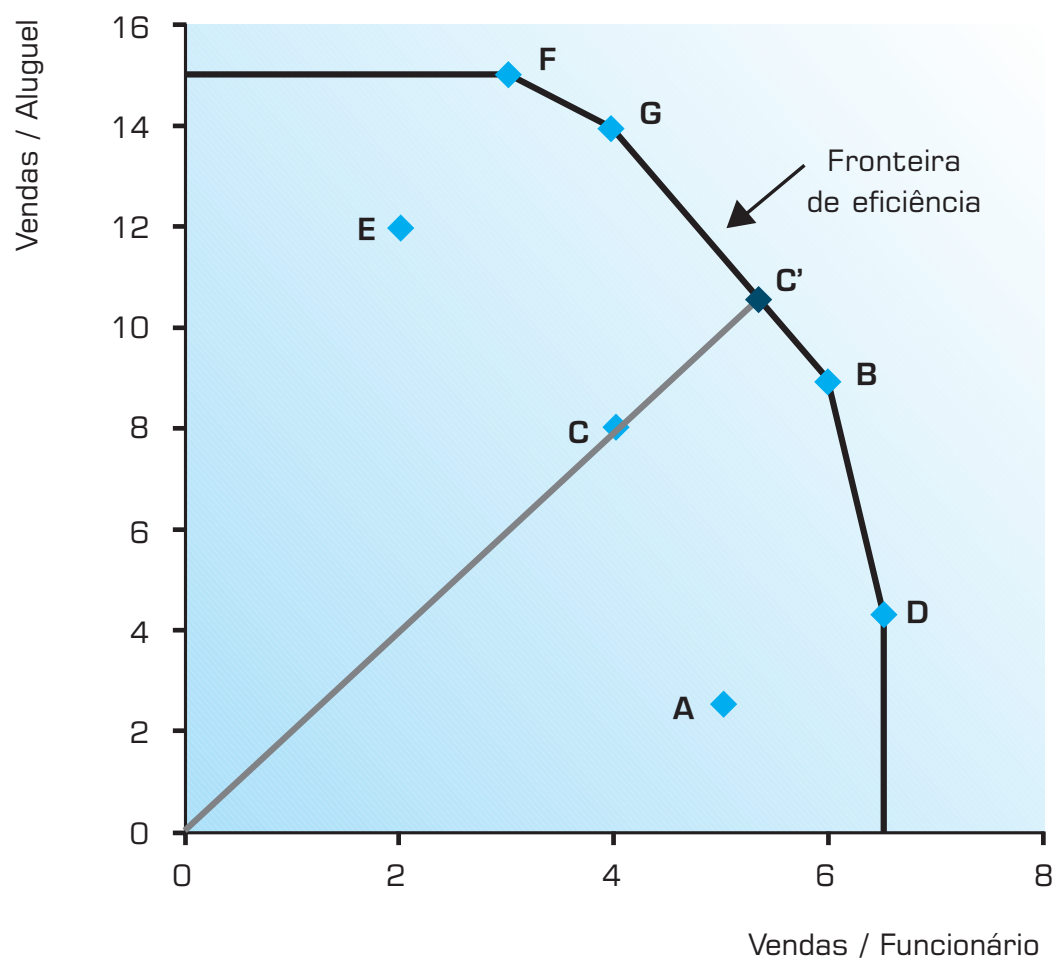


pode ser uma reta, uma parábola etc. Aqui, será utilizado, como exemplo, um arco de circunferência. A circunferência é o lugar geométrico dos pontos eqüidistantes da origem a uma distância comum R da mesma. Estes pontos serão as coordenadas dadas pela razão entre o Produto e os Insumos, como mostrado.

Nossa análise é restrita ao caso de um único Produto, uma situação que não deixa de ser comum. No caso de agências bancárias, por exemplo, apesar de haver diversos produtos que são oferecidos aos clientes (seguro, previdência, cartão de crédito etc.), eles via de regra são analisados independentemente, assim como as metas globais de cada produto são estabelecidas.

\section{A Fronteira de Eficiência Ideal}

Para discutir o que vem a ser uma fronteira de eficiência ideal é preciso relembrar qual é o objetivo perseguido. Desejamos distribuir uma meta total de um Produto para uma rede de DMU's cujos dados de Insumos são conhecidos, de forma não tendenciosa nem arbitrária e de modo que, quando forem avaliadas pelo método DEA, todas as DMU's estejam sobre a fronteira de eficiência. Esta fronteira é gerada por pontos que são a razão entre a meta e cada Insumo. Cada coordenada deste ponto pode ser entendida como a produtividade de uma DMU num aspecto particular (vendas por funcionário, vendas por aluguel etc.), ou seja, na produtividade em relação a algum Insumo.
Não estão registrados neste texto critérios que definam a forma da fronteira de eficiência ideal. Na verdade, é possível que estes critérios nem existam. É preciso, todavia, que essa fronteira seja convexa, de modo a envolver todos os pontos e possíveis combinações lineares dos mesmos. Existem várias funções que atendem a estes requisitos e exibimos algumas delas na Figura 3.

\section{Reta}

Tomar a reta como a forma de uma fronteira de eficiência ideal significa dizer que, para que uma DMU seja eficiente, ela deve ter a soma de suas produtividades (Vendas/Funcionário e Vendas/Aluguel) maximizada. A escolha da reta seria justificável no caso em que todas as DMU's, para serem consideradas igualmente eficientes, devessem ter a soma de suas produtividades igual. A equação da reta é dada por:

$$
x_{1}+x_{2}=\mathrm{B}
$$

em que $B$ é uma constante.

Podemos estabelecer uma relação entre estas produtividades, conjecturando, por exemplo, que a equação da reta seja do tipo:

$a \cdot x_{1}+x_{2}=\mathrm{B}$

sendo $a$ uma constante preestabelecida e $B$ uma constante.

Tabela 2: Eficiências relativas e conjuntos de referência.

\begin{tabular}{|l|c|c|c|c|c|c|c|}
\hline \multicolumn{1}{|c|}{ LOJA } & A & B & G & D & E & F & G \\
\hline Eficiência Relativa & $77 \%$ & $100 \%$ & $78 \%$ & $100 \%$ & $80 \%$ & $100 \%$ & $100 \%$ \\
\hline Conjuntos de Referência & D & & B e G & & F & & \\
\hline
\end{tabular}

Figura 3: Algumas das formas possiveis para a fronteira de eficiência.

Reta

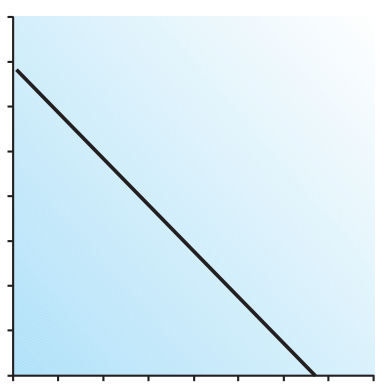

Parábola

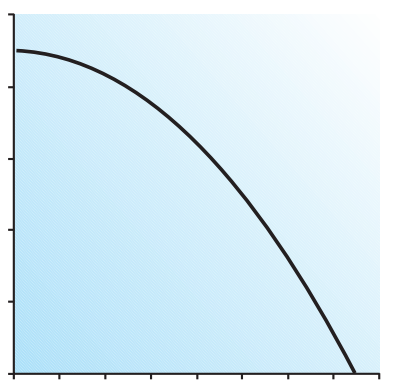

Circunferência

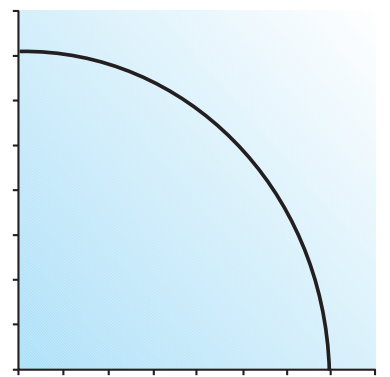

Elipse

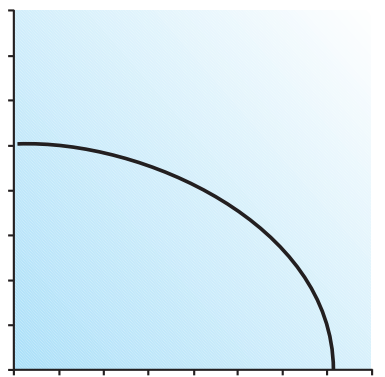




\section{Parábola}

Podemos estabelecer como critério de eficiência para o grupo de DMU's que todas elas tenham como resultado a mesma soma do valor de uma produtividade com o quadrado da outra. Nesse caso, a fronteira de eficiência terá a forma de uma parábola, i.e.:

$$
a \cdot x_{1}+x_{2}^{2}=\mathrm{C}
$$

em que $a$ será preestabelecido e $C$ é uma constante.

\section{Circunferência e Elipse}

A circunferência é o lugar geométrico da soma dos quadrados das coordenadas. Seus pontos internos formam um conjunto convexo. Apesar de não haver justificativas suficientes para sua escolha como a forma ideal, optamos por utilizá-la como exemplo ilustrativo por sua simplicidade e simetria.

Uma vez entendido o processo de cálculo das metas para este caso particular, é possível fazê-lo a partir de outras formas (reta, parábola etc.), cada uma delas com suas características próprias.

A equação da circunferência é dada por:

$$
x_{1}+x_{2}^{2}=R^{2}
$$

Existe, no entanto, o problema de haver Insumos com dimensões distintas, o que exige uma transformação dos dados originais. Como será visto a seguir, a circunferência calculada para os dados transformados se transforma numa elipse, quando os dados iniciais forem novamente postos em cena.

A equação da elipse é dada por:

$$
\frac{x_{1}^{2}}{a_{1}}+\frac{x_{2}^{2}}{a_{2}}=R^{2}
$$

\section{A Fronteira representada por um arco de circunferência}

Utilizando os mesmos dados de Insumos do exemplo anterior, podemos aplicar a metodologia para o cálculo de metas DEA arbitrando como fronteira de eficiência ideal um arco de circunferência. Os dados de Insumo estão reproduzidos na Tabela 3 , a seguir.

Temos, portanto, duas metas para cada loja e nenhum critério para que possamos escolher entre elas. A metodologia DEA tem a propriedade de estabelecer pesos variáveis, não impostos, mas calculados de forma a maximizar a eficiência relativa de cada loja. Estes cálculos asseguram que, se uma DMU estiver sobre a envoltória dos dados, ela terá eficiência $100 \%$. O ideal seria, então, que todas as lojas vendessem de forma a ficar sobre essa fronteira de eficiência. A meta, assim, deve ser esta produção esperada.

Embora não haja como saber, a princípio, onde estará essa fronteira, podemos supor uma forma para ela, respeitada sua condição de convexidade. Como já anunciado, a título de exemplo, utilizaremos um arco de circunferência de raio desconhecido.

Antes de iniciar o equacionamento, porém, notemos que os eixos podem ter dimensões distintas, como no caso do nosso exemplo. Uma alternativa para contornar este problema é fazer um escalonamento dos Insumos, dividindo cada um deles pelo maior valor de seu grupo, para deixá-los adimensionais e entre 0 e 1 .

Sejam $a_{1}$ e $a_{2}$ iguais a $x_{1 G}$ e $x_{2 A}$ (os maiores valores para os Insumos 1 e 2), respectivamente. Obtemos, então, os valores escalonados conforme a Tabela 5 .

Podemos agora calcular a razão entre os dois Insumos Escalonados, razão que define a inclinação das retas sobre as quais os pontos referentes às lojas vão estar.

Tabela 3: Dados das lojas.

\begin{tabular}{|l|c|c|c|c|c|c|c|c|}
\hline \multicolumn{1}{|c|}{ LOJA } & & A & B & C & D & E & F & G \\
\hline Funcionários & $x_{1}$ & 2 & 3 & 4 & 2 & 6 & 5 & 7 \\
\hline Aluguel & $x_{2}$ & 4 & 2 & 2 & 3 & 1 & 1 & 2 \\
\hline
\end{tabular}

Tabela 4: Metas calculadas sob dois critérios.

\begin{tabular}{|l|c|c|c|c|c|c|c|c|}
\hline \multicolumn{1}{|c|}{ LOJA } & A & B & G & D & E & F & G & TOTAL \\
\hline Funcionários & 2 & 3 & 4 & 2 & 6 & 5 & 7 & $\mathbf{2 9}$ \\
\hline Aluguel & 4 & 2 & 2 & 3 & 1 & 1 & 2 & $\mathbf{1 5}$ \\
\hline Meta Funcionários & 6,9 & 10,3 & 13,8 & 6,9 & 20,7 & 17,2 & 24,2 & $\mathbf{1 0 0 , 0 0}$ \\
\hline Meta Aluguel & 26,7 & 13,3 & 13,3 & 20,0 & 6,7 & 6,7 & 13,3 & $\mathbf{1 0 0 , 0 0}$ \\
\hline
\end{tabular}


Todavia, desconhecendo o valor dos Produtos, não é possível, ainda, localizar estes pontos.

Estes Produtos serão as procuradas metas do problema. Em suma, procuramos metas que, se atingidas precisamente pelas DMU's, colocarão todas elas sobre uma fronteira de eficiência dada por uma determinada função convexa, como um arco de circunferência.

$\mathrm{Na}$ Figura 4 exibimos as coordenadas dos valores inversos dos Insumos. Podemos ver estes pontos como o resultado de uma avaliação em que todas as lojas obtiveram Produto igual a 1. Como visto anteriormente, quando somente o Produto varia, o ponto caminha sobre sua reta radial de caminho (pontilhada). O aumento do Produto equivale a um deslocamento do ponto sobre essa reta, se distanciando da origem.

Assim, procuramos os valores que, quando divididos pelos Insumos 1 e 2 de cada loja, levem os respectivos pontos para longe da origem, deixando todos sobre um lugar geométrico predefinido que, como já registramos, arbitramos para efeito ilustrativo como sendo um arco de circunferência com centro na origem. Estes valores procurados devem somar a meta total, preestabelecida. $\mathrm{O}$ raio deste arco ainda é desconhecido, porém não arbitrário pois, como veremos, está definido por condições de contorno.

Para demonstrar a obtenção deste raio matematicamente definimos as seguintes variáveis:

$x_{1 j}$ é o valor do Insumo 1 da loja $j=1, \ldots, 7$.

$x_{2 j}$ é o valor do Insumo 2 da loja $j=1, \ldots, 7$.

$a_{1}$ é o valor máximo do Insumo 1 (neste caso $a_{1}=x_{1 G}$ ).

$a_{1}$ é o valor máximo do Insumo 2 (neste caso $a_{2}=x_{2 A}$ ).

$\lambda_{1 j}=\frac{a_{1}}{x_{1 j}} \quad \begin{aligned} & \text { é o valor da abscissa da loja } j=1, \ldots, 7 \\ & \text { para Produto }=1 .\end{aligned}$

$\lambda_{2 j}=\frac{a_{2}}{x_{2 j}} \quad \begin{aligned} & \text { é o valor da ordenada da loja } j=1, \ldots, 7 \\ & \text { para Produto }=1 .\end{aligned}$

$\alpha_{j}$ é o coeficiente angular da reta que passa pela origem e pelo ponto $\left(\lambda_{1 j}, \lambda_{2 j}\right), j=1, \ldots, 7$.

Figura 4: Retas de caminho das lojas.

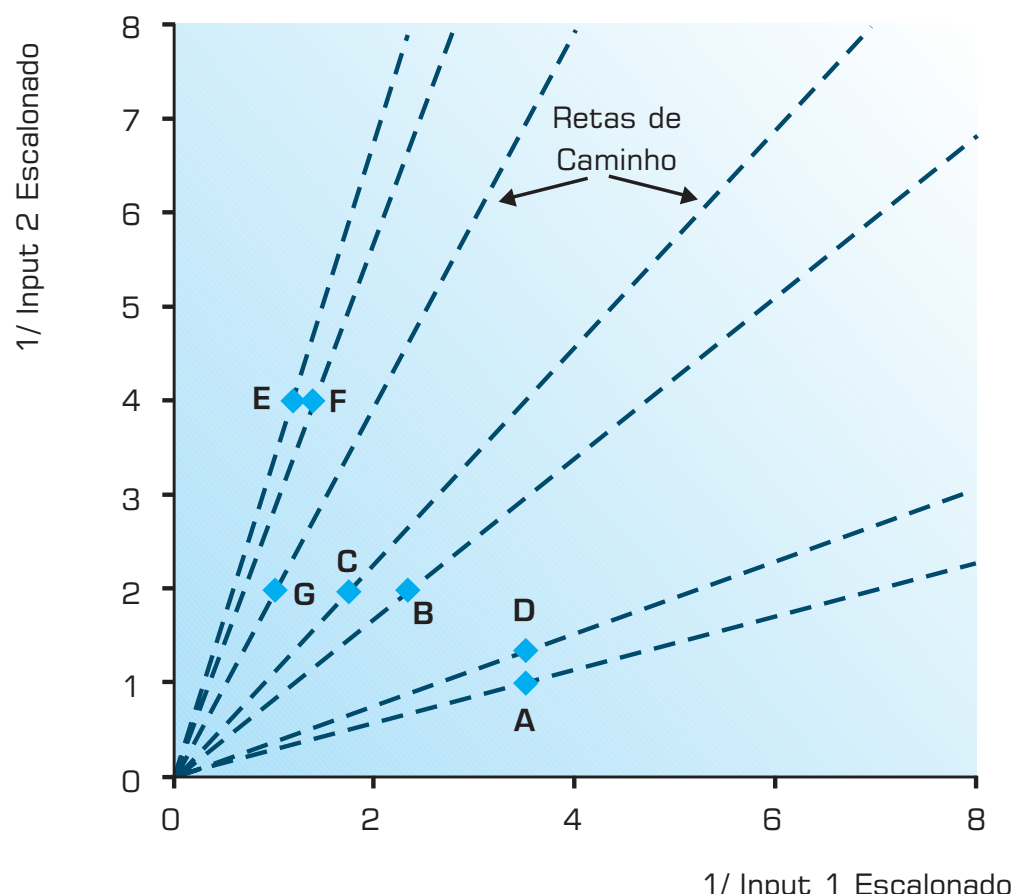

Tabela 5: Dados das lojas escalonados.

\begin{tabular}{|c|c|c|c|c|c|c|c|c|}
\hline LOJA & & A & B & C & D & E & F & G \\
\hline Insumo 1 Escalonado & $x_{1} / a_{1}$ & 0,29 & 0,43 & 0,57 & 0,29 & 0,86 & 0,71 & 1,00 \\
\hline Insumo 2 Escalonado & $x_{2} / a_{2}$ & 1,00 & 0,50 & 0,50 & 0,75 & 0,25 & 0,25 & 0,50 \\
\hline
\end{tabular}


$M_{j}$ é o valor da meta de cada loja $j=1, \ldots, 7$.

$\lambda_{1 j}{ }^{\prime}=\frac{a_{1}}{x_{1 j}} \cdot M_{j} \quad \begin{aligned} & \text { é o valor da abscissa da loja } j=1, \ldots, 7 \\ & \text { para Produto }=M_{j}\end{aligned}$

$\lambda_{2 j}{ }^{\prime}=\frac{a_{2}}{x_{2 j}} \cdot M_{j} \quad \begin{aligned} & \text { é o valor da ordenada da loja } j= \\ & 1, \ldots, 7 \text { para Produto }=M_{j}\end{aligned}$

$M_{T}$ é a Meta Total para o grupo de lojas.

A Meta Total é a soma das metas individuais das lojas. Então:

$$
M_{T}=\sum_{j=1}^{7} M_{j}=\sum_{j=1}^{7} \frac{\lambda_{1 j}{ }^{\prime} \cdot x_{1 j}}{a_{1}}=\sum_{j=1}^{7} \frac{\lambda_{2 j}{ }^{\prime} \cdot x_{2 j}}{a_{2}}=100
$$

Os coeficientes angulares das retas são dados por:

$\alpha_{j}=\frac{\lambda_{2 j}}{\lambda_{1 j}}=\frac{\lambda_{2 j}{ }^{\prime}}{\lambda_{1 j}{ }^{\prime}}$

A circunferência de raio $R$ será coincidente com todos os pontos $\left(\lambda_{1 j}{ }^{\prime}, \lambda_{2 j}{ }^{\prime}\right)$, de modo que:

$$
\begin{aligned}
& R^{2}=\left(\lambda_{1 j}{ }^{\prime}\right)^{2}+\left(\lambda_{2 j}{ }^{\prime}\right)^{2}, j=1, \ldots, 7 \\
& R^{2}=\left(\lambda_{1 j}{ }^{2}\right)^{2}+\left(\alpha_{j} \cdot \lambda_{1 j}{ }^{\prime}\right)^{2} \\
& R=\lambda_{1 j} \cdot \sqrt{1+\alpha_{j}^{2}}
\end{aligned}
$$

Esta equação possui duas incógnitas. Para encontrar o valor de $\mathrm{R}$, é preciso isolar $\lambda_{1 j}{ }^{\prime}$ :

$$
\lambda_{1 j}^{\prime}=\frac{R}{\sqrt{1+\alpha_{j}^{2}}}
$$

Da equação (1), temos:

$$
M_{T}=\sum_{j=1}^{7} M_{j}=\sum_{j=1}^{7} \frac{\lambda_{1 j}{ }^{\prime} \cdot x_{1 j}}{a_{1}}=\sum_{j=1}^{7} \frac{R}{\sqrt{1+\alpha_{j}^{2}}} \cdot \frac{x_{1 j}}{a_{1}}
$$

O raio da fronteira de eficiência fica dado por:

$$
R=\frac{M_{T} \cdot a_{1}}{\sum_{j=1}^{7} \frac{x_{1 j}}{\sqrt{1+\alpha_{j}^{2}}}}
$$

E as metas de cada loja são calculadas por:

$$
M_{j}=\lambda_{1 j} \cdot \frac{x_{1 j}}{a_{1}}=\frac{R}{\sqrt{1+\alpha_{j}{ }^{2}}} \cdot \frac{x_{1 j}}{a_{1}}, \mathrm{j}=1, \ldots 9
$$

Utilizando os dados para as 7 lojas e após realizar todos os cálculos, obtemos $R=46,16$

Figura 5: Fronteira de eficiência ideal como um arco de circunferência.

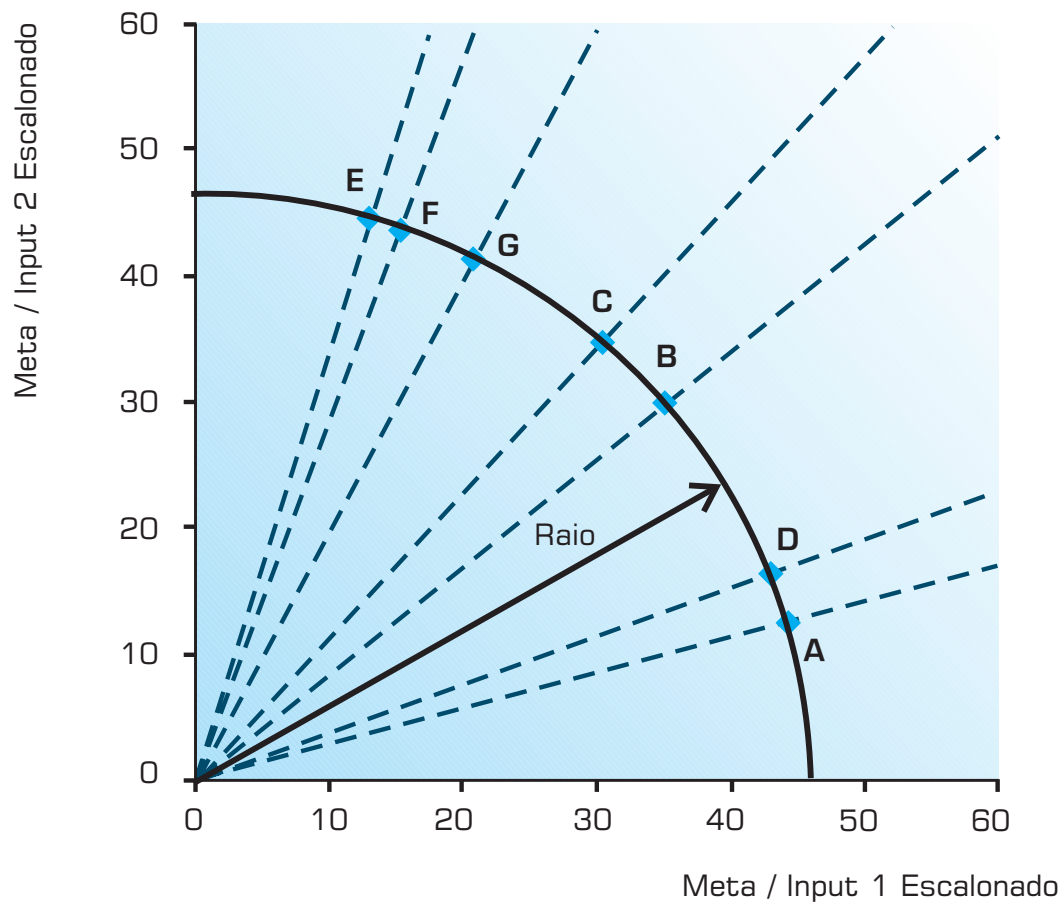


Na Tabela 6, exibimos as metas obtidas para cada uma das lojas e, na Figura 5, mostramos os pontos referentes às 7 lojas.

Os valores das coordenadas horizontais e verticais são a Meta dividida por Insumo 1 Escalonado e Insumo 2 Escalonado.

Imaginemos que as Metas fossem rigorosamente cumpridas por todas as 7 lojas. Os resultados finais seriam os que estão expressos na Tabela 7.

Se plotarmos a figura da Fronteira de Eficiência com os valores de Meta/Funcionário e Meta/Aluguel, perceberemos que ela deixará de ser uma circunferência e se transformará numa elipse, já que as grandezas são diferentes. A fronteira de eficiência real será como a mostrada na Figura 6 e todas as lojas terão eficiência relativa $100 \%$.

Tabela 6: Metas calculadas.

\begin{tabular}{|c|c|c|c|c|c|c|c|c|}
\hline LOJA & A & B & C & D & E & F & G & TOTAL \\
\hline Meta Calculada & 12,68 & 15,02 & 17,37 & 12,32 & 11,08 & 10,89 & 20,64 & 100,00 \\
\hline
\end{tabular}

Tabela 7: Resultados esperados.

\begin{tabular}{|l|r|r|r|r|r|r|r|}
\hline \multicolumn{1}{|c|}{ LOJA } & A & B & G & D & E & F & G \\
\hline Funcionários & 2 & 3 & 4 & 2 & 6 & 5 & 7 \\
\hline Aluguel & 4 & 2 & 2 & 3 & 1 & 1 & 2 \\
\hline Vendas = Metas & 12,68 & 15,02 & 17,37 & 12,32 & 11,08 & 10,89 & 20,64 \\
\hline Vendas / Funcionário & 6,34 & 5,01 & 4,34 & 6,16 & 1,85 & 2,18 & 2,95 \\
\hline Vendas / Aluguel & 3,17 & 7,51 & 8,68 & 4,11 & 11,08 & 10,89 & 10,32 \\
\hline
\end{tabular}

Figura 6: Fronteira de eficiência ideal (Elipse).

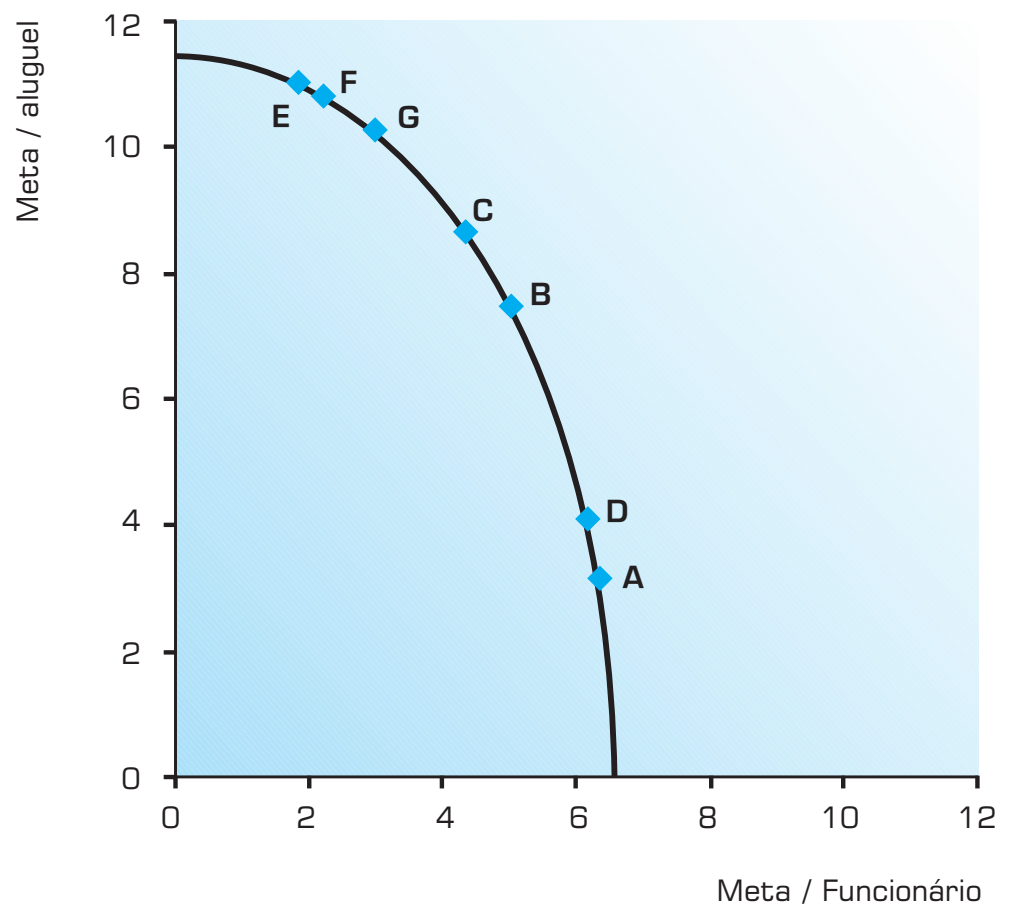




\section{Dados com Dimensões Diferentes}

Umas das características da análise DEA é o fato de ela ser feita sem a preocupação com as dimensões dos dados. Podemos ter, por exemplo, como Insumos, populações de países e PIB per capita [LINS et al., 2003]. A dimensão dos valores de um Insumo em relação à dos outros Insumos não é importante, já que o problema de maximização estabelece pesos variáveis que compensam essa discrepância de grandezas. No entanto, a forma da envoltória de dados resultante será fruto dessas discrepâncias. Assim, no estabelecimento de metas, este fato deve ser considerado, pois estamos partindo da forma da fronteira de eficiência para chegar aos valores das metas, e não ao contrário, que é o procedimento ortodoxo. Estas metas, portanto, serão conseqüência da forma escolhida.

Podemos encontrar uma fórmula que expressa a Meta de uma loja diretamente a partir dos dados do problema [KOZYREFF, 2002].

$$
M_{j}=\frac{M_{T}}{\sqrt{\frac{a_{1}^{2}}{x_{1 j}^{2}}+\frac{a_{2}^{2}}{x_{2 j}^{2}}} \cdot \sum_{j=1}^{n} \frac{1}{\sqrt{\frac{a_{1}^{2}}{x_{1 j}^{2}}+\frac{a_{2}^{2}}{x_{2 j}^{2}}}}}
$$

A partir da fórmula encontrada observamos que se os valores de $x_{2 j}$ forem muito maiores do que os valores de $x_{1 j}$, eles quase não farão efeito no cálculo da meta, já que estão elevados à potência -2. As somas existentes dentro das duas raízes serão muito mais afetadas pelos valores do Insumo 1.

Por esta razão, é necessário analisar a ordem de grandeza dos Insumos. O arco de circunferência só foi admitido desde que o maior valor dos dois Insumos fosse igual a 1 , e por isso foi feito um escalonamento destes dados.

Após calcularmos as metas e fazermos a análise DEA admitindo Metas = Vendas, verificamos que a fronteira de eficiência resultante, quando inseridos os dados dos Insumos não escalonados, tomou a forma de uma elipse. A relação entre os raios maior e menor da elipse é a igual à relação entre o maior valor de Insumo $1\left(a_{2}=x_{1 G}=7\right) \mathrm{e}$ o maior valor do Insumo $2\left(a_{1}=x_{2 A}=4\right)$.

Não necessariamente devemos utilizar o valor máximo de um Insumo como parâmetro para a forma da fronteira ideal, até porque estes valores podem estar absurdamente acima dos outros. Outra alternativa seria usar a média como parâmetro. Esta discussão, porém, não será abordada neste trabalho e fica proposta como assunto de investigação futura.

\section{MÚLTIPLOS INSUMOS E UM PRODUTO}

O raciocínio para 3 Insumos é igual ao feito para 2, substituindo a reta por um plano, a parábola por um parabolóide, o arco de circunferência por um segmento de casca esférica, e assim por diante.

O objetivo desta seção é demonstrar como o caso de 3 Insumos pode ser generalizado a partir da reprodução da lógica empregada no caso de 2 Insumos. Para problemas com mais de 3 Insumos, embora o desenho da fronteira não seja mais possível, os cálculos para a determinação das metas são possíveis e análogos.

Todo o desenvolvimento apresentado a seguir é baseado numa fronteira elíptica no $\mathfrak{R}^{m}$ ( $m$ é o número de DMU's), sendo os coeficientes da elipse os maiores valores de cada Insumo.

Relembrando o conceito de eficiência relativa dado por DEA, temos que:

$$
E_{j}=\frac{u_{1} y_{1 j}+u_{2} y_{2 j}+\ldots+u_{s} y_{s j}}{v_{1} x_{1 j}+v_{2} x_{2 j}+\ldots+v_{m} x_{m j}}
$$

em que:

$E_{j}$ é a Eficiência Relativa da DMU j

$u_{i}$ é o peso dado ao Produto i

$y_{i j}$ é a quantidade de Produto i da unidade $\mathrm{j}$

$v_{i}$ é o peso dado ao Insumo $\mathrm{i}$

$x_{i j}$ é a quantidade de Insumo i da unidade $\mathrm{j}$

Considerando o caso de apenas um Produto, a equação (7) será simplificada para:

$$
E_{j}=\frac{u y_{j}}{\sum_{i=1}^{m} v_{i} x_{i j}}
$$

O objetivo, aqui, é estabelecer metas de vendas para DMU's. Feito isto, se todas elas alcançarem estas metas, terão eficiência $100 \%$. Podemos renomear o numerador e o denominador da expressão acima, respectivamente, como a Produção e a Meta de uma DMU:

$$
P_{j}=u y_{j} \quad M_{j}=\sum_{i=1}^{m} v_{i} x_{i j}
$$

E a eficiência, então, será dada pela razão entre a Produção e a Meta da DMU:

$$
E_{j}=\frac{P_{j}}{M_{j}}
$$

Os dados do problema são os $m$ Insumos $x_{i}$ das $n$ DMU's. 
Para proceder com os cálculos, façamos uma transformação de coordenadas:

$$
\lambda_{i j}=\frac{a_{i}}{x_{i j}}, x_{i j}>0
$$

em que: $a_{i}=\operatorname{MAX}\left(x_{i 1}, \ldots, x_{i j}, \ldots, x_{i n}\right)$

A restrição acima indica que não pode haver Insumos nulos. Casos em que isto ocorre devem ser vistos particularmente. Pode-se, como alternativa, substituir os zeros pelo menor valor do Insumo dentre as DMU's. A Tabela 9 apresenta as coordenadas das DMU's.

Assim, cada DMU será dada por um ponto $\mathrm{DMU}_{j}\left(\lambda_{1 j}\right.$, $\left.\lambda_{2 j}, \ldots, \lambda_{m j}\right)$ no $\mathfrak{R}^{m}$.

Estes pontos, naturalmente, estão sobre retas que os ligam à origem. Procuramos os pontos $\mathrm{DMU}_{j}\left(\lambda_{1 j}\right.$, , $\left.\lambda_{2 j}, \ldots, \lambda_{m j}{ }^{\prime}\right)$ sobre estas mesmas retas, mas que também pertençam à fronteira de eficiência.

As coordenadas $\lambda_{i j}$ e $\lambda_{i j}$ ' guardam a seguinte relação:

$$
\lambda_{i j}{ }^{\prime}=\theta_{j} \cdot \lambda_{i j} \theta_{j} \in \mathfrak{R} \text { e } j=1,2, \ldots, n
$$

A fronteira de eficiência atende à seguinte equação:

$$
\lambda_{1 j}{ }^{\prime 2}+\lambda_{2 j}{ }^{\prime 2}+\ldots+\lambda_{m j}{ }^{\prime 2}=R^{2}, \quad j=1,2, \ldots, n
$$

O valor de $\theta_{j}$ não é nada mais do que a própria Meta para cada DMU, pois o que estamos procurando são números que, quando multiplicados por $\lambda_{i i j}$ coloquem os pontos sobre a fronteira de eficiência Ideal.
Estes pontos são as coordenadas $\left(\frac{M_{j}}{x_{1 j}}, \frac{M_{j}}{x_{2 j}}, \ldots, \frac{M_{j}}{x_{m j}},\right)$ de todas as $j$ DMU's.

Temos então que:

$$
\frac{M_{j}}{x_{i j}}=\frac{M_{j}}{\frac{a_{i}}{\lambda_{i j}}}=\frac{\lambda_{i j} \cdot M_{j}}{a_{i}}=\frac{\lambda_{i j}{ }^{\prime}}{a_{i}}
$$

E, portanto,

$$
\mathrm{M}_{j}=\theta_{j}
$$

A Meta Total é a soma de todas as Metas individuais:

\begin{tabular}{|c|c|c|c|c|}
\hline $\mathbf{i} \quad \mathbf{j}$ & DMU, $_{1}$ & $\mathbf{D M U}_{2}$ & $\cdots$ & $\mathbf{D M U}_{\mathrm{n}}$ \\
\hline$x_{1}$ & $x_{11}$ & $x_{12}$ & $\ldots$ & $x_{1 n}$ \\
\hline$x_{2}$ & $x_{21}$ & $x_{22}$ & $\cdots$ & $x_{2 n}$ \\
\hline$\cdots$ & $\cdots$ & $\cdots$ & $\ldots$ & $\cdots$ \\
\hline$x_{m}$ & $x_{m 1}$ & $x_{m 2}$ & $\cdots$ & $x_{m n}$ \\
\hline
\end{tabular}
$\sum_{j=1}^{n} M_{j}=M_{T}$

Combinando as equações, temos:

\begin{tabular}{|c|c|c|c|c|}
\hline $\mathbf{i} \mathbf{j}$ & $\mathrm{DMU}_{1}$ & $\mathrm{DMU}_{2}$ & $\cdots$ & $\mathbf{D M U}_{n}$ \\
\hline$\lambda_{1}$ & $\lambda_{11}$ & $\lambda_{12}$ & $\ldots$ & $\lambda_{1 \mathrm{n}}$ \\
\hline$\lambda_{2}$ & $\lambda_{21}$ & $\lambda_{22}$ & $\ldots$ & $\lambda_{2 n}$ \\
\hline$\ldots$ & $\ldots$ & $\ldots$ & $\cdots$ & $\ldots$ \\
\hline$\lambda_{m}$ & $\lambda_{\mathrm{m} 1}$ & $\lambda_{\mathrm{m} 2}$ & $\cdots$ & $\lambda_{\mathrm{mn}}$ \\
\hline
\end{tabular}

$$
\begin{aligned}
\sum_{i=1}^{m} \lambda_{i j}{ }^{2}=\sum_{i=1}^{m} M_{j}{ }^{2} \cdot \lambda_{i j}{ }^{2} & =M_{j}{ }^{2} \sum_{i=1}^{m} \lambda_{i j}{ }^{2}=R^{2}, j=1,2, \ldots, n \\
M_{j} & =\frac{R}{\sqrt{\sum_{i=1}^{m} \lambda_{i j}^{2}}}
\end{aligned}
$$

Tabela 8: Dados de Insumos das DMU's.

Tabela 9: Dados escalonados invertidos. 
Lembrando que $\lambda_{i j}=\frac{a_{i}}{x_{i j}}$, obtém-se:

$$
M_{j}=\frac{R}{\sqrt{\sum_{i=1}^{m} \frac{a_{i}{ }^{2}}{x_{i j}^{2}}}}
$$

O valor de $R$ ainda é incógnito. Para calculá-lo, façamos o desenvolvimento abaixo:

$$
\begin{gathered}
\sum_{j=1}^{n} M_{j}=R \cdot \sum_{j=1}^{n} \frac{1}{\sqrt{\sum_{i=1}^{m} \lambda_{i j}^{2}}}=M_{T} \\
M_{T}=R \cdot \sum_{j=1}^{n} \frac{1}{\sqrt{\sum_{i=1}^{m} \frac{a_{i}^{2}}{x_{i j}^{2}}}}
\end{gathered}
$$

Desta forma, $\mathrm{R}$ é dado diretamente a partir dos dados iniciais do problema.

$$
R=\frac{M_{T}}{\sum_{j=1}^{n} \frac{1}{\sqrt{\sum_{i=1}^{m} \frac{a_{i}^{2}}{x_{i j}^{2}}}}}
$$

Finalmente, podemos calcular todas as metas da DMU diretamente dos dados iniciais:

$$
M_{j}=\frac{M_{T}}{\sqrt{\sum_{i=1}^{m} \frac{a_{i}^{2}}{x_{i j}^{2}}} \cdot \sum_{j=1}^{n} \frac{1}{\sqrt{\sum_{i=1}^{m} \frac{a_{i}^{2}}{x_{i j}^{2}}}}}
$$

A equação acima é em tudo semelhante à apresentada para o caso com 2 Insumos, representando bem a analogia estabelecida na generalização de procedimentos do caso de 2 para 3 Insumos.

\section{EXTENSÕES}

A única hipótese do método desenvolvido neste trabalho foi a de que a fronteira DEA ideal tem a forma de um arco de circunferência. Assim, fica como sugestão a aplicação do método a outros perfis de fronteira que sejam considerados apropriados segundo um embasamento teórico específico. Por fim, fica também como sugestão de continuidade o desenvolvimento de uma metodologia para os casos com múltiplos produtos, assunto que não foi abordado neste trabalho.

\section{Artigo recebido em 18/12/2002 Aprovado para publicação em 12/08/2004}

\section{- Referências Bibliográficas}

ALVES, R.T.T. "Desenvolvimento de uma metodologia de avaliação de agências bancárias baseada em Análise de Envoltória de Dados (DEA)". Trabalho de Graduação Instituto Tecnológico de Aeronáutica, São José dos Campos, SP, 130 p., 2001.

AVELLAR, J.V.G.; POLEZZI, A.O.D.; e MILIONI A.Z. "On the evaluation of Brazilian landline telephone services companies", Pesquisa Operacional, v. 22, Num. 2, 231-246, 2002
BANKER R. D.; CHARNES C.; COOPER W.W. "Some models for estimating technical and scale inefficiencies in Data Envelopment Analysis", Management Science, v. 30, n. 9, p. 1.078-1.092, 1984

CHARNES A.; COOPER W.W; LEWIN A.Y. and SEIFORD, L. "Data Envelopment Analysis: theory, methodology and applications". Norwell, Massachusets, EUA: Kluwer Academic Publishers, 512 p. 1.994 .
CHARNES A.; COOPER W.W. e RHODES E. "Measuring the efficiency of decision making units", European Journal of Operational Research, v 2, p. 429-444, 1978.

COOPER, W.; SEIFORD, L.; TONE, K. "Data Envelopment Analysis: a comprehensive text with models, applications, references and DEA-solver software". Norwell, Massachusets, EUA: Kluwer Academic Publishers, 307 p. 2.000.

LINS, M.P.E., GOMES, E.G., SOARES DE MELLO, J.C.C.B., SOARES DE MELLO, A.J.R. "Olympic ranking based on a Zero Sum Gains DEA model". European Journal of Operational Research, v. 148 , n. 2, p. 312-322, 2003.

KOZYREFF, E. "Um Método para Estimativa de Metas DEA-Eficientes" Trabalho de Graduação - Instituto Tecnológico de Aeronáutica, São José dos Campos, SP. 50 p., 2002.

SILVA, F. M. "Um estudo sobre Data Envelopment Analysis", Trabalho de Graduação - Instituto Tecnológico de Aeronáutica, São José dos Campos, SP. 64 p., 2000. 